\title{
A natural antisense transcript, BOKAS, regulates the pro-apoptotic activity of human Bok
}

\author{
HONG ZHANG, SHUPING GAO ${ }^{1}$ and CHRISTIAN DE GEYTER \\ University Women's Hospital Basel and Research Department of University Hospital, \\ Hebelstrasse 20, CH-4031 Basel, Switzerland
}

Received October 6, 2008; Accepted December 11, 2008

DOI: 10.3892/ijo_00000241

\begin{abstract}
Bok is a proapoptotic member in the Bcl-2 family and the expression of Bok is regulated by cellular stresses. In the present study, we have isolated and characterized a natural antisense transcript of Bok, BOKAS. The BOKAS gene consists of 2 exons, that expresses as a non-protein coding transcript. Both BOKAS and Bok are transcribed from the same locus but in opposite orientations. The mRNA expression of BOKAS was only detected in testis and certain cancer tissues but not in other normal adult tissues examined. Overexpression of BOKAS alone exhibited no significant anti- or pro-apoptotic activity but it was able to inhibit Bokinduced apoptosis in HeLa cells. Our results suggest that BOKAS may function specifically in the human testis, where it serves as an antisense molecule to regulate Bok-induced apoptosis. In addition, natural antisense transcripts were identified for BAD and BCL2L12.
\end{abstract}

\section{Introduction}

Bcl-2 family proteins are essential regulators of apoptosis and play an important role in embryonic development and in adult tissue homeostasis. Dysregulation of apoptosis is involved in the development of many diseases such as cancer, autoimmunity and various degenerative diseases (reviewed in refs. 1 and 2). Bok is one of the pro-apoptotic members consisting of BH1-3 domains $(3,4)$. It has been demonstrated that the expression of hBok could be regulated by cis- and trans-acting elements (5-8).

Natural antisense transcripts are RNAs possessing transcript complementarity to other RNA transcripts within cells (9).

Correspondence to: Dr Hong Zhang, University Women's Hospital Basel and Research Department of University Hospital, Hebelstrasse 20, CH-4031 Basel, Switzerland

E-mail: hzhang@uhbs.ch

Present address: ${ }^{1}$ Human Stem Cell Research Lab, Geneva University Hospital, 30 Bld. De la Cluse, 1211 Geneva, Switzerland

Key words: hBok, BOKAS, natural antisense transcripts, testis
These RNAs can be either protein-coding or non-coding RNAs (10). It has been shown that natural antisense transcripts are important factors involved in gene regulation (9). Moreover, aberrant natural antisense transcription might contribute to major diseases, such as $\alpha$-thalassemia, Parkinson's disease and some malignant cancers (11).

During our study of Bok expression, we identified a novel natural antisense transcript of Bok, denominated BOKAS. We demonstrated that it is a negative regulator of Bok-induced apoptosis being expressed only in testis and in some cancer cells.

\section{Materials and methods}

Cloning and chromosomal location of BOKAS. During our study of Bok expression, we found several alternative splicing variants of hBok by RT-PCR. The full length nucleotide sequence of Bok was then extensively subjected to homology search using the NCBI BLASTN algorithm (12) against the human EST database. Among the sequences identified, one novel sequence (IMAGE: 6173204) was found to contain nucleotide sequences with significant identity to that of Bok but in opposite orientation. Full length cDNA was assembled after 5'RACE and was termed BOKAS. The GenBank accession no. for BOKAS is FJ185168. For mammalian expression, the BOKAS cDNA was cloned in the NotI/SalI site of pSPORT VI. The genomic organization of BOKAS was found according to the BLAT:UCSC Human Genome Browser (Mar 2006 release).

Tissue expression pattern analysis by RT-PCR. RT-PCR was performed using the primers 5'-GTTCTGGCTTCAGGATC TGC-3' and 5'-TCCAAAATAGGCCTCAGTGG -3' on a panel of first-strand cDNAs from various human tissues (BD). The PCR products should be $245 \mathrm{bp}$ in size and were sequenced.

Cell culture and cell viability assay. HeLa cells were seeded at $5 \times 10^{4}$ cells in 12 -well plates with $10 \%$ FBS. Cells were transfected using Superfect (Qiagen, Hilden, Germany) with $2 \mu \mathrm{g}$ of plasmids of total in all settings. pcDNA3.Bok:GFP was transfected with pSPORT VI- BOKAS in the ratio of $1: 1 \mu \mathrm{g}$ and 0.5:1.5 $\mu \mathrm{g}$. Green fluorescent cells were monitored, both normal flat and detached apopotic cells were counted by microscopic examination after $48 \mathrm{~h}$ of serum deprivation. 
A

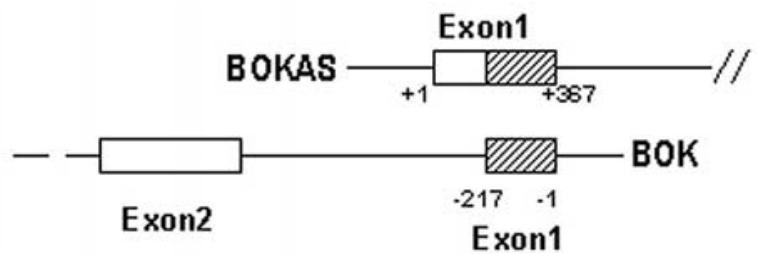

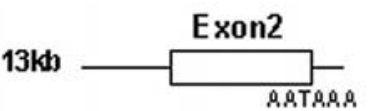

B

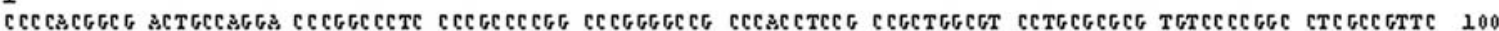

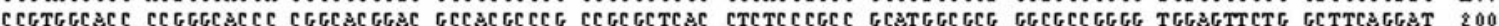

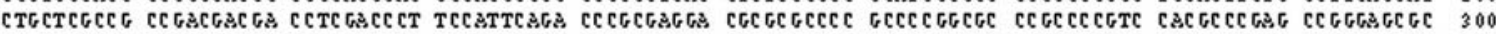
CAG GTCCACG GGGCTTCCCG CGCTCTTGG GGGCTCCTGC CGCGGCGCG GGGCCTGGG CAGC GAGGC CTCCAGAGG ACCAGCGCTG CCGACTCCTT 400

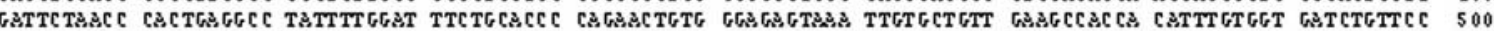

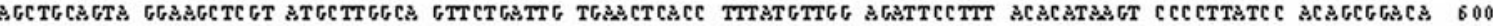
AATTC CATGA сC CCAGTG ATGC CTGAA TCGCG GA GA TACTGATCC TACATATAAT GTGTTCTTTC CTATATGTAT ATGTATATAT CTATATCTAT

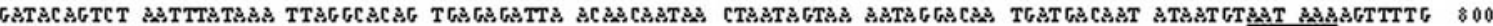
TGA.ATATGGT CTCTCTTCTC TCTC 2222222 22222222222 2222222222

C

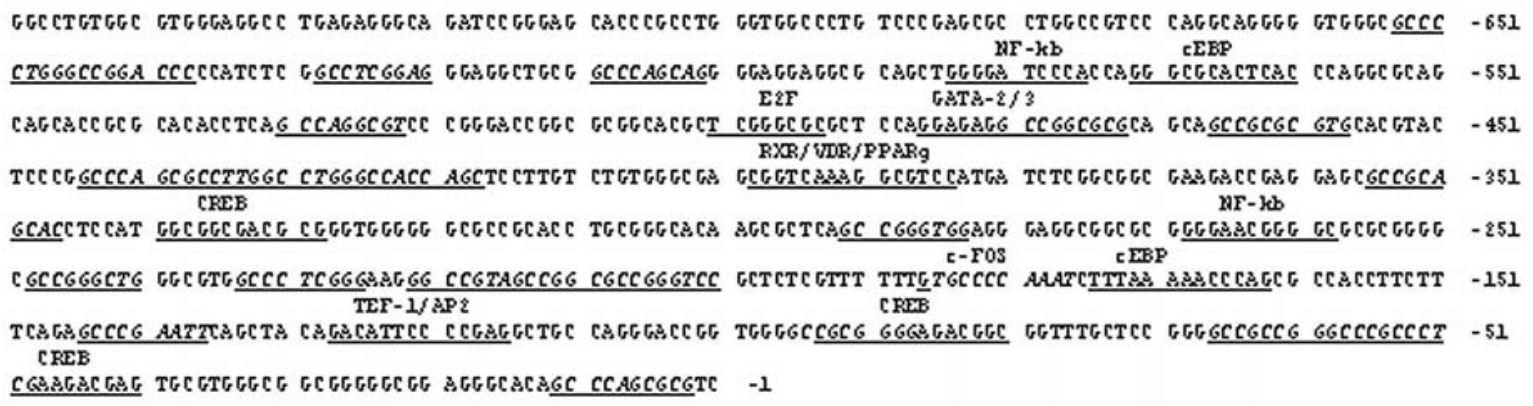

Figure 1. Identification of cDNAs for a novel human BOKAS. (A) Schematic representation of genomic organization of BOKAS in the relation with hBok. Exon regions are denoted by boxes. The black horizontal lines denote the introns. Black boxes indicate the regions with $100 \%$ identity in both genes but opposite orientation (B). The nucleotide sequence of BOKAS cDNA (Genebank accession no. FJ185168) demonstrate that it is a non-protein coding transcript. (C) The promoter region of BOKAS. Underlines represent transcriptional factor binding sites.

At least 100 green cells from each individual culture were analysed. Apoptosis is expressed as percentage of dying cells per total number of cells, given with the standard deviation of the assay (13).

\section{Results and Discussion}

BOKAS is a natural antisense transcript of the hBok gene. We have found several alternative splicing variants of hBok by RT-PCR (data not shown). Further screening of the GenBank ${ }^{\mathrm{TM}}$ ESTs database with the full length Bok nucleotide sequence led to isolation of a novel transcript that contains a 217-bp long sequence of Bok but in opposite orientation (Fig. 1A). The direction of transcription was examined through the PCR evaluation with primers spanned the intron-exon splice junctions (see Materials and methods). It was then termed BOKAS and considered as a natural antisense transcript of hBok gene (with GenBank accession no. FJ185168, Fig. 1B). The full-length BOKAS transcript has 855 nucleotides but does not contain a long open reading frame, giving 79 amino acids in conceptually translation, and we were not able to detect any signals using a coupled transcription-translation system (data not shown). Together, the results suggested that BOKAS is a non-protein coding transcript. Searching the BLAT:UCSC Human Genome Browser (Mar 2006 release) revealed that the BOKAS cDNA sequence matched exactly to the genomic sequence in chromosome 2 and it contains 2 exons. Part of the first exon matched with $100 \%$ identity that of the Bok gene in opposite directions. The second exon located some $13 \mathrm{~kb}$ away from the 5'UTR of Bok. The polyadenylation signal (AATAAA) was detected 30-bp upstream of the poly(A) (Fig. 1B). These results indicate that both BOKAS and Bok are transcribed from the same locus but in opposite orientations and that BOKAS represents a cis-natural antisense transcripts (Fig. 1A). Prediction of $750 \mathrm{bp}$ in the promoter/enhancer region was preformed with program Matrix Search on upstream of the transcription start site (http://rulai.cshl.edu/cgi-bin/TRED/tred.cgi?process=home). Several transcription factor binding sites were predicted such as c-Fos and NF- $\mathrm{\kappa B}$ binding elements (Fig. 1C). Strikingly, 28 AP-2 binding sites have been found with some overlapping by CREB, TEF-1 and cEBP.

BOKAS mRNA is differentially expressed in human tissues. We have used a semiquantitive PCR-based system to profile BOKAS mRNA expression in 24 different human organ tissues by using a pair of primers corresponding to sequences flanking to the two exons (Materials and methods). In contrast to the expression of Bok mRNA in colon, stomach, testis, placenta, pancreas, ovary and uterus (5), BOKAS expression could only be detected in the testis (Fig. 2). The study from the EST database revealed that BOKAS was also expressed 


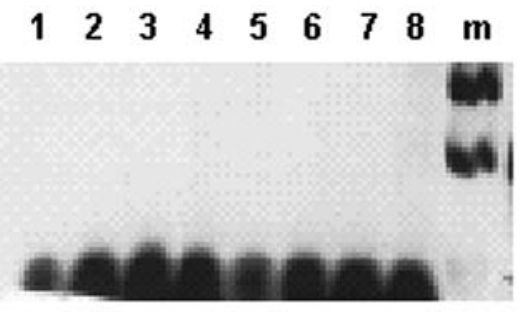

\section{$\begin{array}{lllllllll}9 & 10 & 11 & 12 & 13 & 14 & 15 & 16 & \mathrm{~m}\end{array}$}

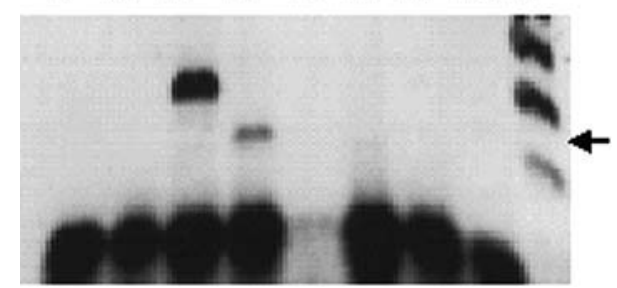

Figure 2. The expression of BOKAS mRNA in different tissues. PCR was performed on first strand cDNAs prepared from various human tissues (BD human multiple tissue cDNA panels). The numbers indicate tissues examined, 1 (heart), 2 (brain), 3 (placenta), 4 (lung), 5 (liver), 6 (muscle), 7 (kidney), 8 (pancreas), 9 (spleen), 10 (thymus), 11 (prostate), 12 (testis), 13 (ovary), 14 (small intestine), 15 (colon), 16 (peripheral blood leukocyte) and $\mathrm{m}$ (markers).

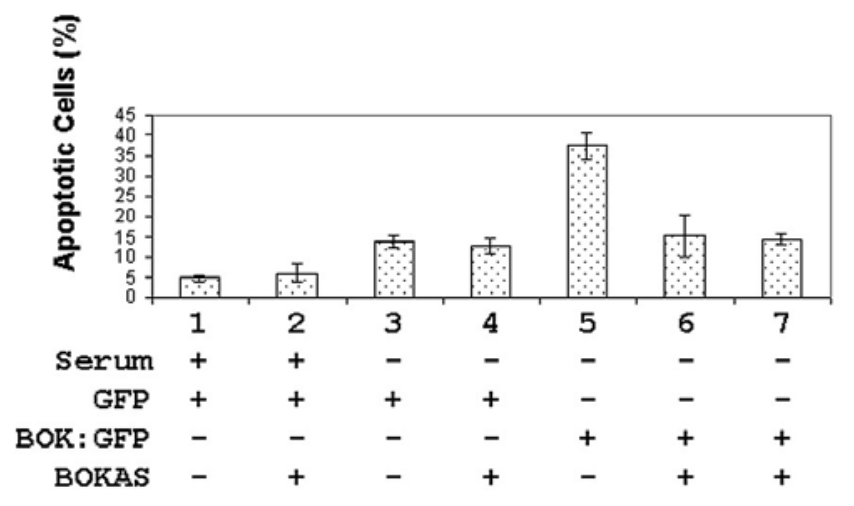

Figure 3. Suppression of Bok-induced apoptosis by BOKAS in HeLa cells. HeLa cells were cotransfected as pSPORT VI- BOKAS with plasmid pEGFPC1 encoding GFP or pSPORT VI- BOKAS or pcDNA3-Bok:GFP carrying the genes for BOKAS together with Bok. After $48 \mathrm{~h}$ of serum deprivation, normal flat (survival) and round apoptotic cells were counted. Apoptosis is expressed (in \%) as percentage of dying cells per total number of cells. Six is the transfectant with pcDNA3.Bok:GFP and pSPORT VI-BOKAS in the ratio of $1: 1 \mu \mathrm{g}$. Seven indicates the ratio of 0.5:1.5 $\mu \mathrm{g}$.

in fetal tissues and certain cancer cells, such as, fetal brain (EST AL534911, EST CR619343), and leiomyosarcoma uterus (BU184197). In addition, we also observed a longer PCR product in prostate. However, we could not confirm that the extra band corresponds to an alternative transcript since the sequence from the PCR product did not match to any known genomic sequences (data not shown) and sequences at the Chr.2 3q37.3 region have not yet been fully sequenced.

Overexpression of BOKAS reduced Bok-induced cell death. To assess the effect of BOKAS on Bok-induced apoptosis, we overexpressed ectopically BOKAS in HeLa cells. As shown in Fig. 3, expression of BOKAS alone exhibited no
A

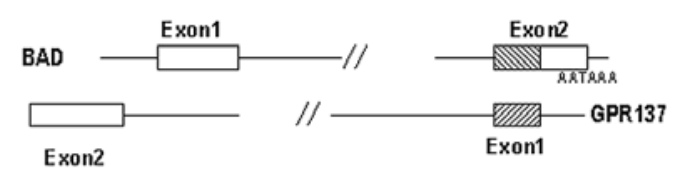

B

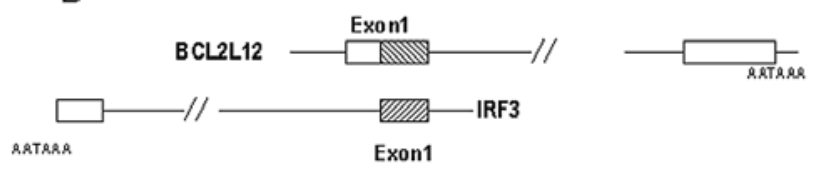

Figure 4. Natural antisense transcripts within the BCL2 family. Schematic representation of genomic organization of BAD in the relation with GPR137 (A) and BCL2L12 to interferon regulatory factor 3 (IRF3, B). Exon regions are denoted by boxes. The black horizontal lines denote the introns. Black boxes indicate the regions with $100 \%$ identity in both genes but opposite orientation.

significant anti- or pro-apoptotic activity when compared with control plasmids (pEGFP-C1). Forty-eight hours after transfection/serum deprivation, the number of apoptotic cells transfected with the control plasmid were $13 \%$ as compared to $<5 \%$ of cells with serum in culture, whereas the expression of Bok:GFP encoding Bok-GFP fusion proteins (13) increased the number of apoptosis to $\sim 40 \%$, indicating that Bok expression sensitized cells to become apoptosis upon serum deprivation. The BOKAS construct transfected together with Bok reduced apoptotic cells close to $15 \%$. These results indicated that BOKAS is a negative regulator of apoptosis induced by Bok.

Natural antisense transcripts within the BCL2 family. BOKAS is the first natural antisense transcript described in the $\mathrm{Bcl} 2$ family. The results prompted us to study in silico if any other natural antisense transcripts could be discovered in the Bcl2 family. Nucleotide sequences of full length and their alternative splicing variants were used to search the NCBI ESTs database, the results revealed that natural antisense transcripts could be identified for BAD and BCL2L12 (Fig. 4). BAD is gene encoding a $\mathrm{BH} 3$-only pro-apoptotic protein (14). Several EST clones (CN431725 from kidney cancer cells, BX415776 and CF541292) have been found to contain sequences of BAD 3'UTR in opposite directions. Sequence analysis revealed that these sequences code for putative $G$ protein-couple receptor 137 with unknown function. BCL2L12 is an anti-apoptotic member of the family (15). Its exon I sequence matched in an opposite strand to numerous ESTs, such as DA559544 from heart and DB123802 from thymus. BLAST studies indicated that these clones are transcripts of interferon regulatory factor 3 (16). Thus 5' UTR of interferon regulatory factor 3 contains the natural antisense sequence matched to part of 5' UTR and encoding region of BCL2L12.

Taken together, all results describe a novel natural antisense transcript, BOKAS, being testis-specific and inhibiting Bok-induced apoptosis. Increasing numbers of natural antisense transcripts have been identified in various species $(11,17)$. They play a potential role for gene regulation through several mechanisms. It remains to be determined whether BOKAS regulates expression of Bok via retention of Bok in the 
nucleus by forming dsRNA duplexes or via degradation of Bok mRNA or through other mechanisms. Bok expression was detected in human, mouse and rat testis. The testisspecific expression of BOKAS would affect the known role of Bok during testicular development $(5,18)$. Moreover, natural antisense transcripts were found in other members of the $\mathrm{Bcl} 2$ family, indicating that natural antisense transcripts may be regulatory factors in modulating apoptosis via the BCL2 family. Since they are present in cancer cells, it could be informative to examine whether upregulation of antisense transcripts in tumor cells would further enhance their malignancy.

\section{Acknowledgements}

We are grateful to The HUGO Gene Nomenclature Committee for providing the nomenclature of BOK antisense RNA (nonprotein coding), BOKAS.

\section{References}

1. Adams JM and Cory S: Life-or-death decisions by the Bcl-2 protein family. Trends Biochem Sci 26: 61-66, 2001.

2. Gross A, McDonnell JM and Korsmeyer SJ: Bcl-2 family members and the mitochondria in apoptosis. Genes Dev 13: 1899-1911, 1999.

3. Zhang H, Holzgreve W and De Geyter C: Evolutionarily conserved Bok proteins in the Bcl-2 family. FEBS Lett 480: 311-313, 2000 .

4. Hsu SY, Kaipia A, McGee E, et al: Bok is a pro-apoptotic Bcl-2 protein with restricted expression in reproductive tissues and heterodimerizes with selective anti-apoptotic Bcl-2 family members. Proc Natl Acad Sci USA 94: 12401-12406, 1997.

5. Gao S, Fu W, Dürrenberger M, et al: Membrane translocation and oligomerization of $\mathrm{hBok}$ are triggered in response to apoptotic stimuli and Bnip3. Cell Mol Life Sci 62: 1015-1024, 2005.
6. Rodriguez JM, Glozak MA, Ma Y, et al: Bok, Bcl-2-related ovarian killer, is cell cycle-regulated and sensitizes to stressinduced apoptosis. J Biol Chem 281: 22729-22735, 2006.

7. Yakovlev AG, Di Giovanni S, Wang G, et al: BOK and NOXA are essential mediators of p53-dependent apoptosis. J Biol Chem 279: 28367-28374, 2004.

8. Soleymanlou N, Wu Y, Wang JX, et al: A novel Mtd splice isoform is responsible for trophoblast cell death in pre-eclampsia. Cell Death Differ 12: 441-452, 2005.

9. Osato N, Suzuki Y, Ikeo K, et al: Transcriptional interferences in cis-natural antisense transcripts of humans and mice. Genetics 176: 1299-1306, 2007.

10. Lavorgna G, Dahary D, Lehner B, et al: In search of antisense. Trends Biochem Sci 29: 88-94, 2004

11. Zhang Y, Liu XS, Liu QR, et al: Genome-wide in silico identification and analysis of cis natural antisense transcripts (cis-NATs) in ten species. Nucleic Acids Res 3: 3465-3475, 2006.

12. Lennon G, Auffray C, Polymeropoulos M, et al: The I.M.A.G.E. Consortium: an integrated molecular analysis of genomes and their expression. Genomics 33: 151-152, 1996.

13. Zhang H, Cowan-Jacob SW, Simonen M, et al: Structural basis of BFL-1 for its interaction with BAX and its antiapoptotic action in mammalian and yeast cells. J Biol Chem 275: 11092-11099, 2000.

14. Yang E, Zha J, Jockel J, Boise LH, Thompson CB and Korsmeyer SJ: Bad, a heterodimeric partner for Bcl-XL and Bcl-2, displaces Bax and promotes cell death. Cell 80: 285-291, 1995.

15. Scorilas A, Kyriakopoulou L, Yousef GM, Ashworth LK, Kwamie A and Diamandis EP: Molecular cloning, physical mapping, and expression analysis of a novel gene, BCL2L12, encoding a proline-rich protein with a highly conserved $\mathrm{BH} 2$ domain of the Bcl-2 family. Genomics 72: 217-221, 2001.

16. Servant MJ, Grandvaux N and Hiscott J: Multiple signaling pathways leading to the activation of interferon regulatory factor 3. Biochem Pharmacol 64: 985-992, 2002.

17. Werner A: Natural antisense transcripts. RNA Biol 2: 53-62, 2005.

18. Suominen JS, Yan W, Toppari J, et al: The expression and regulation of Bcl-2-related ovarian killer (Bok) mRNA in the developing and adult rat testis. Eur J Endocrinol 145: 771-778, 2001. 